\title{
The Effect of Intravenous Magnesium Sulfate and Lidocaine in Hemodynamic Responses to Endotracheal Intubation in Elective Coronary Artery Bypass Grafting: A Randomized Controlled Clinical Trial
}

\author{
Mehrdad Mesbah Kiaee ${ }^{1}$; Saeid Safari ${ }^{1}$; Gholam Reza Movaseghi ${ }^{1}$; Mahmoud Reza \\ Mohaghegh Dolatabadi ${ }^{1}$; Masoud Ghorbanlo ${ }^{1}$; Mehrnoosh Etemadi ${ }^{2}$; Seyed Arash Amiri ${ }^{1}$; \\ Mohammad Mahdi Zamani ${ }^{1,2,}$ \\ ${ }^{1}$ Department of Anesthesiology and Pain Medicine, Iran University of Medical Sciences, Tehran, Iran \\ ${ }^{2}$ Students' Scientific Research Centre, Tehran University of Medical Sciences, Tehran, Iran \\ ${ }^{*}$ Corresponding author: Mohammad Mahdi Zamani, Department of Anesthesiology and Pain Medicine, Iran University of Medical Sciences, Tehran, Iran. Tel/Fax: +98-2188989162, \\ E-mail:mmzses@gmail.com
}

Received: November 1, 2013; Revised: December 21, 2013; Accepted: January 8, 2014

\begin{abstract}
Background: There have been many concerns about alteration in hemodynamic parameters within and shortly after endotracheal intubation (ETI) in patients scheduled for elective coronary artery bypass grafting (CABG).

Objectives: We compared the attenuation effect of magnesium sulfate and lidocaine on hemodynamic responses after ETI, in patients undergoing CABG.

Patients and Methods: In this randomized controlled trial 150 patients undergoing elective CABG were enrolled. Included patients were randomly allocated to three groups and received lidocaine $(1.5 \mathrm{mg} / \mathrm{kg})$, magnesium sulfate $(50 \mathrm{mg} / \mathrm{kg}$ within five minute), or normal saline, 90 seconds before ETI. Baseline hemodynamic parameters including heart rate(HR), systolic blood pressure(SBP), diastolic blood pressure (DBP), and mean arterial pressure (MAP) were recorded immediately before anesthesia induction, before intubation, immediately after intubation, and at second and fifth minutes after intubation.

Results: The baseline hemodynamic variables had no deference among the three groups. HR between intubation and five minute after intubation was significantly lower in two groups received lidocaine or magnesium sulfate in comparison with placebo group. Lidocaine induced more than 20\% decrease in HR and MAP immediately after intubation; hence, lidocaine group showed significant MAP reduction in comparison with the two other groups.

Conclusions: Lidocaine induced hemodynamic instability but premedication of magnesium sulfate maintained hemodynamic stability after intubation. Therefore, in patients undergoing CABG who received high-dose intravenous analgesic for general anesthesia, the administration of magnesium sulfate might result in maintaining hemodynamic stability after ETI in comparison with lidocaine.
\end{abstract}

Keywords: Coronary Artery Bypass Grafting; Endotracheal Intubation; Hemodynamic; Lidocaine; Magnesium Sulfate

\section{Background}

The inappropriate response of hemodynamic parameters to endotracheal intubation (ETI) can increase perioperative and postoperative morbidity and mortality, particularly in patients with cardiovascular disease or those undergoing coronary artery interventions (1). A variety of medications have been suggested to control hemodynamic responses and prevent hemodynamic instabilities. In this regard, the beneficial effects of adding magnesium sulfate (MGS) to anesthetic drugs on improving intraoperative conditions such as minimizing hemodynamic instability were hypothesized (2). MGS inhibits catecholamines release from adrenal medulla and peripheral nerve endings and directly blocks catecholamines receptors. Therefore, MGS causes sympathetic block and indirectly causes dilated blood vessels and finally, reduces blood pressure (BP) (3). Studies on animals and human subjects have shown that the addition of intrathecal MGS may reduce the incidence of side effects observed after local anesthesia (4). Furthermore, intrathecal MGS injection would result in reducing postoperative pain and prolonged sensory blockade duration $(5,6)$. It has also been suggested that intravenous (IV) MGS can inhibit ETIassociated catecholamine release with that may result in stability of postoperative hemodynamic status (7). Moreover, MGS attenuates vasopressin-stimulated vasoconstriction and normalizes sensitivity to vasopressin (8). 
On the other hand, protective effects of IV lidocaine on intraoperative or postoperative hemodynamic changes are still unknown. Although some experimental studies could not show changes in hemodynamic condition following injection of lidocaine in anesthetized subjects (9), some others have emphasized the attenuation of cardiovascular responses to ETI by this drug (10).

\section{Objectives}

In the present study, we compared the attenuation effect of magnesium sulfate and lidocaine on hemodynamic responses after ETI in patients undergoing coronary artery interventions.

\section{Patients and Methods}

\subsection{Study Design}

In this randomized controlled double-blind study, we recruited 150 patients scheduled for elective coronary artery bypass grafting (CABG) under general anesthesia with ETI at Moheb Hospital, a private referral general hospital in Tehran, Iran. Those with American Society of Anesthesiologists (ASA) class more than II, obesity (body mass index > 30), difficulty in ETI, emergency surgery, left ventricular ejection fraction of less than $45 \%$ based on echocardiography reports, cardiac arrhythmias, renal dysfunction (serum creatinine level $>2 \mathrm{mg} / \mathrm{dL}$ ), history of hypersensitivity to lidocaine or MGS, and pregnant women were excluded. The study protocol was approved by the Ethics Committee of Moheb Hospital and informed consents were obtained from all patients. Patients were randomly allocated to three groups that received lidocaine (1.5 mg/kg), MGS (50 mg/kg within 5 minutes), or normal saline. Routine monitoring of electrocardiogram, pulse oximetry, non-invasive and invasive BP monitoring from left radial artery were commenced at operation room before surgery. For all the patients, IV line was secured with gauge-18 catheter and 3 to $4 \mathrm{~mL} / \mathrm{kg}$ of isotonic crystalloid fluid was infused to check the IV line efficacy. Then the assigned drugs were administered intravenously 90 seconds before ETI. The syringes were filled with one of the study medications, namely, MGS, lidocaine, or normal saline (placebo), by an anesthesiology technician and handed over to the anesthesiologist who was blinded to the medications. Therefore, none of the patients and their anesthesiologists know the group that patients were allocated to.

\subsection{Anesthesia Technique}

Anesthetic management of patients in all study groups was similar. All patients were premedicated by intramuscular morphine $(0.1 \mathrm{mg} / \mathrm{kg})$ half an hour before surgery and ringer lactate infusion ( 8 to $10 \mathrm{~mL} / \mathrm{kg}$ ) was started. After preoxygenation with fraction of inspired oxygen $\left(\mathrm{FIO}_{2}\right)$ of 1.0 , anesthesia was induced with sufentanil
(0.5-1 $\mu \mathrm{g} / \mathrm{kg})$, midazolam (20 $\mu \mathrm{g} / \mathrm{kg})$, etomidate (0.15-0.3 $\mathrm{mg} / \mathrm{kg}$ ), and cisatracurium (0.15-0.2 $\mathrm{mg} / \mathrm{kg}$ ). After three minutes, patients were intubated with the appropriate size, cuffed endotracheal tube (ETT). All intubations were performed by an experienced anesthesiologist. Anesthesia was maintained with infusion of midazolam (0.25-0.5 $\mu \mathrm{g} / \mathrm{kg} / \mathrm{min}$ ) and fentanyl (0.03-0.1 $\mu \mathrm{g} / \mathrm{kg} / \mathrm{min})$ and bolus administration of cisatracurium $(0.03 \mathrm{mg} / \mathrm{kg})$ every 30 minutes required. In all study groups, ventilation was stopped during the period of cardiopulmonary bypass. At the end of bypass, lungs were manually reinflated under direct observation using a continuous positive airway pressure CPAP of $20 \mathrm{~cm} \mathrm{H}_{2} \mathrm{O}$.

\subsection{Study Measurements}

Hemodynamic parameters of patients including systolic BP (SBP), diastolic BP (DBP), mean arterial pressure (MAP), and heart rate (HR) were recorded immediately before anesthesia induction, before ETI, immediately after ETI, and at second and fifth minutes after ETI.

\subsection{Study Definitions}

Hemodynamic stability was determined as changes between $-20 \%$ to $+20 \%$ from baseline BP. Diagnosis of hypertension was determined by positive history of antihypertensive drugs use. Positive history of cigarette smoking was defined as smoking cigarette for more than ten pack-years.

\subsection{Statistical Analysis}

According to the similar previous study on 60 patients (30 in each group), we determined that a sample size of about 50 in each group would be sufficient to detect the difference in hemodynamic indices by considering a standard deviation of $20 \%$ of mean difference as the minimum detectable difference of means hemodynamic indices among three groups, a power of $95 \%$, and a significance level of $5 \%$.

All statistical analyses were performed using SPSS software (version 20.0, SPSS Inc., Chicago, IL, the USA). Data were expressed as mean \pm standard deviation for quantitative variables and were summarized by absolute frequencies and percentages for categorical variables. Categorical variables were compared using Chisquare test or Fisher's exact test. Quantitative variables were also compared with one-way ANOVA test. Differences in the trend of changes in hemodynamic parameters among study groups were examined using the repeated measure ANOVA test. Statistical significance was determined as a P value $<0.05$

\section{Results}

Demographic data were similar among three groups (Table 1). The baseline hemodynamic parameters were not different among three groups $(\mathrm{P}>0.05)$. Table 2 represents 
Mesbah Kiaee M et al.

the hemodynamic parameters of three groups at the study time points. HR was significantly lower from ETI time through the fifth minute after ETI in those receiving lidocaine or MGS in comparison with the placebo group. There were no trends in the hemodynamic parameters in comparison to the baseline values in MGS group; all parameters were reduced from anesthesia induction through ETI time, then elevated after ETI by about 10\% from baseline, and then gradually decreased until the fifth minutes after ETI (Table 2). The trends of changes in the parameters between placebo and MGS groups were not significantly different. Inter-group comparison showed significantly lower DBP and MAP immediately after ETI in the lidocaine group in comparison with two other groups.

\begin{tabular}{|c|c|c|c|c|}
\hline Characteristics & Control Group $(\mathrm{No}=52)$ & Lidocaine Group $(\mathrm{No}=48)$ & $\operatorname{MgSO4}$ Group $(\mathrm{No}=50)$ & Pvalue \\
\hline Sex, Male/Female & $32 / 20$ & $32 / 16$ & $34 / 16$ & 0.769 \\
\hline Age, $y$ & $59.2 \pm 12.2$ & $60.1 \pm 5.4$ & $58.6 \pm 8.6$ & 0.770 \\
\hline BMI, $\mathrm{kg} / \mathrm{m}^{2}$ & $26.4 \pm 4.3$ & $25.7 \pm 4.7$ & $24.8 \pm 4.0$ & 0.199 \\
\hline Ejection Fraction,\% & $48.0 \pm 4.6$ & $49.3 \pm 9.0$ & $49.5 \pm 5.7$ & 0.462 \\
\hline Valvular Heart Disease & $0(0.0)$ & $3(6.3)$ & $2(4.0)$ & 0.209 \\
\hline Myocardial Infarction & $12(23.1)$ & $11(22.9)$ & $10(20.0)$ & 0.916 \\
\hline Hypertension & $32(61.5)$ & $30(62.5)$ & $27(54.0)$ & 0.640 \\
\hline Diabetes Mellitus & $16(30.8)$ & $20(42.6)$ & $18(36.0)$ & 0.476 \\
\hline Smoking & $10(19.2)$ & $8(16.7)$ & $12(24.0)$ & 0.653 \\
\hline \multicolumn{5}{|l|}{ Medication } \\
\hline Beta-Blocker & $51(98.1)$ & $44(91.7)$ & $44(88.0)$ & 0.141 \\
\hline Calcium-Blocker & $18(34.6)$ & $17(35.4)$ & $17(34.0)$ & 0.989 \\
\hline ACE-I & $35(67.3)$ & $30(62.5)$ & $36(72.0)$ & 0.605 \\
\hline Nitrates & $42(80.8)$ & $38(79.2)$ & $40(80.0)$ & 0.980 \\
\hline
\end{tabular}

a Abbreviations: BMI, body mass index; and ACE-I, angiotensin converting enzyme-inhibitor.

${ }^{b}$ Data are presented as mean \pm SD or No. $(\%)$.

Table 2. Hemodynamic Status During Study ${ }^{\mathrm{a}}$

\begin{tabular}{|c|c|c|c|c|}
\hline Characteristic & Placebo Group $(\mathrm{No}=52)$ & Lidocaine group $(\mathrm{No}=48)$ & MgSO4 Group $($ No = 50) & Pvalue \\
\hline \multicolumn{5}{|c|}{ Systolic Blood Pressure, $\mathrm{mmHg}$} \\
\hline Before Induction & $152.6 \pm 23.7$ & $160.2 \pm 24.8$ & $154.7 \pm 22.8$ & 0.266 \\
\hline Before Intubation & $100.5 \pm 20.4$ & $97.7 \pm 16.9$ & $115.5 \pm 25.3$ & 0.437 \\
\hline After Intubation & $130.7 \pm 34.0$ & $119.9 \pm 30.2$ & $120.6 \pm 34.5$ & 0.095 \\
\hline 2 min After Intubation & $146.5 \pm 13.0$ & $129.2 \pm 28.9$ & $126.4 \pm 26.8$ & 0.398 \\
\hline 5 min After Intubation & $113.3 \pm 18.4$ & $111.4 \pm 24.0$ & $112.5 \pm 19.5$ & 0.896 \\
\hline \multicolumn{5}{|c|}{ Diastolic Blood Pressure, mmHg } \\
\hline Before Induction & $78.7 \pm 12.4$ & $77.1 \pm 10.6$ & $77.5 \pm 10.5$ & 0.756 \\
\hline Before Intubation & $55.6 \pm 10.0$ & $53.0 \pm 8.8$ & $66.2 \pm 9.2$ & 0.161 \\
\hline After Intubation & $74.3 \pm 17.4$ & $66.7 \pm 14.2$ & $70.9 \pm 18.9$ & 0.018 \\
\hline 2 min After Intubation & $67.8 \pm 12.0$ & $64.2 \pm 12.6$ & $66.0 \pm 13.9$ & 0.388 \\
\hline 5 min After Intubation & $64.0 \pm 9.4$ & $61.0 \pm 10.6$ & $64.2 \pm 10.6$ & 0.148 \\
\hline \multicolumn{5}{|c|}{ Mean Arterial Pressure, $\mathrm{mmHg}$} \\
\hline Before Induction & $104.5 \pm 18.0$ & $106.2 \pm 16.2$ & $105.2 \pm 14.8$ & 0.870 \\
\hline Before Intubation & $70.1 \pm 13.2$ & $68.6 \pm 12.3$ & $77.7 \pm 11.6$ & 0.606 \\
\hline After Intubation & $92.4 \pm 23.9$ & $82.4 \pm 20.9$ & $84.4 \pm 24.4$ & 0.049 \\
\hline 2 min After Intubation & $86.2 \pm 17.7$ & $81.4 \pm 17.1$ & $81.2 \pm 19.1$ & 0.238 \\
\hline 5 min After Intubation & $79.9 \pm 12.3$ & $77.7 \pm 13.8$ & $78.4 \pm 14.3$ & 0.702 \\
\hline \multicolumn{5}{|l|}{ Heart Rate, beat/min } \\
\hline Before Induction & $82.6 \pm 17.7$ & $79.9 \pm 14.7$ & $70.5 \pm 15.5$ & 0.413 \\
\hline Before Intubation & $69.0 \pm 14.0$ & $66.2 \pm 12.0$ & $65.7 \pm 13.0$ & 0.384 \\
\hline After Intubation & $80.9 \pm 16.0$ & $74.3 \pm 14.3$ & $75.3 \pm 15.2$ & 0.048 \\
\hline 2 min After Intubation & $77.5 \pm 14.7$ & $71.7 \pm 14.5$ & $71.1 \pm 13.4$ & 0.046 \\
\hline 5 min After Intubation & $73.8 \pm 13.8$ & $68.3 \pm 13.2$ & $67.6 \pm 11.8$ & 0.034 \\
\hline
\end{tabular}




\section{Discussion}

The present study revealed that in patient undergoing CABG who had received high-dose IV analgesic for general anesthesia, the administration of MGS resulted in maintaining hemodynamic stability after ETI. These effects were significant in comparison with lidocaine, which induced more than $20 \%$ reduction in SBP, DBP, and MAP, and placebo, which caused more increase in SBP, DBP, and MAP; however, both Lidocaine and MGS may lead to similar reduction in HR after ETI. The mechanism of the action of both drugs is obviously multifactorial. The different possible mechanisms of action of MGS have been discussed. It was reported that MGS can induce endothelium-derived nitric oxide production that mediates the relaxation of vascular smooth muscles through its vasodilatory effect (11). In addition, MGS acts as a vasodilator by increasing the synthesis of prostacyclin as well as inhibiting angiotensin converting enzyme activity (12). The mechanism of action is unclear, but its blocking effects on calcium channels and N-methyl-Daspartate (NMDA) receptors seems to play an important role $(12,13)$.

In this study, the MGS group received 50-mg/kg MGS as an IV bolus in a five-minute period before the anesthesia induction. This regimen resulted in a steady and smooth reduction in MAP and reduced HR with no episodes of severe hypotension, which is similar to previous studies $(14,15)$. MGS was chosen since it is a vasodilator with minimal myocardial depression (16) which is the dosedependent depressant effect on cardiac contractility. It has been shown that the depressant effect of MGS on cardiac function is offset by lowering the systemic vascular resistance (SVR) and hence, MGS maintains cardiac pump function (17). We know that MAP is determined by cardiac output (CO), SVR, and central venous pressure (CVP) according to the following equation, which is based on the association among flow, pressure, and resistance: $\mathrm{MAP}=(\mathrm{CO} \times \mathrm{SVR})+\mathrm{CVP} ; \mathrm{CVP}$ is usually at or near $0 \mathrm{mmHg}$; therefore, this formula is often simplified to: MAP $\sim \mathrm{CO} \times \mathrm{SVR}$. Hence, changes in either CO or SVR will affect MAP. In Khajavi et al. study on 32 major nonlaparoscopic gastrointestinal surgeries, premedication with $40 \mathrm{mg} / \mathrm{kg}$ bolus and $10 \mathrm{mg} / \mathrm{kg}$ intraoperative infusion of MGS decreased both intraoperative CO and SVR in comparison with placebo and thus, MAP decreased during operation in MGS group while increased in placebo group $(\mathrm{P}<0.001)(18)$. Shin et al. investigated the lower bolus dose of MGS (10 and $20 \mathrm{mg} / \mathrm{kg}$ ) prior to muscle relaxant and detected the attenuating effect of MGS on rocuronium injection-associated pain as well as laryngoscopy and ETI-associated cardiovascular changes (19). In addition, the role of preoperative MGS administration in controlling intraoperative hypertension and reducing the intraoperative variability of arterial pressure has been studied in patients with hypertension undergoing cataract surgery with local anesthesia (20).
It has been shown that MGS, as a safe drug without any hemodynamic instability, is as effective as nicardipine in controlling arterial pressure during cardiac procedures (21) and shortens postoperative time for extubation in elective CABG surgeries (22). Some previous studies have demonstrated that the infusion of 1.5 to $2 \mathrm{mg} / \mathrm{kg}$ of lidocaine from the fifth to the second minute before laryngoscopy can blunt the increase in HR, SBP, MAP and catecholamine levels associated with intubation (23-25). Another studies found that IV lidocaine with similar dosages failed to control the hemodynamic response following laryngoscopy and ETI (26). This controversy may be referred to the importance of timing of the lidocaine administration. Considering the mechanism of lidocaine, inhibiting the sympathetic response associated with tracheal stimulation appears to result from an increased threshold for airway stimulation, central inhibition of sympathetic transmission, and direct depression of cardiovascular responses (27).

In a recent study by Panda et al., patients with hypertension undergoing elective surgery under general anesthesia were studied. A total of 80 patients were randomly allocated to three groups of MGS infusion at dose of 30,40, or $50 \mathrm{mg} / \mathrm{kg}$ before induction of anesthesia, and a group of $1.5 \mathrm{-mg} / \mathrm{kg}$ lidocaine bolus 90 seconds before intubation. MAP was maintained within normal limits with 30 -mg/kg MGS while 40 and $50 \mathrm{mg} / \mathrm{kg}$ of MGS induced a significant decrease in MAP. A total of six patients with 40 $\mathrm{mg} / \mathrm{kg}$ and ten patients with $50 \mathrm{mg} / \mathrm{kg}$ of MGS required interventions. Only one patient with lidocaine required intervention. On the other hand, anyone with $30-\mathrm{mg} / \mathrm{kg}$ MGS required intervention. Panda et al. concluded that 30-mg/kg MGS was better than lidocaine administration in patients with hypertension and regarding dose of MGS, a further step-up in the dose of MGS from 30 to $50 \mathrm{mg} / \mathrm{kg}$ might cause significant hypotension and more medical expenses (28). According to our findings, administration of MGS helps to maintain BP at the lower limit of normal without adverse effect on BP or HR. Regarding the effects of lidocaine on BP responses, our study showed that the administration of lidocaine $(1.5 \mathrm{mg} / \mathrm{kg})$ before intubation resulted in decreased BP and HR in comparison with placebo. Nooraei et al. showed similar results but they concluded that MGS might increase the HR (29). Although both MGS and lidocaine might reduce HR, MGS is preferred because of its beneficial effects on maintaining BP after intubation in CABG; moreover, MGS was safer than lidocaine in maintaining BP stability after ETI.

Patients suffering from cardiovascular disease, especially those who are candidate for CABG, need hemodynamic stability and MGS stabilizes their BP more effectively than lidocaine. For such patients, anesthesia induction with high-dose IV analgesic would lead to BP suppression while MGS attenuate this effect. Lidocaine induces more than $20 \%$ suppression from baseline after one minute of ETI while MGS provides hemodynamic stability during the five minutes after ETI. 


\section{Acknowledgements}

The authors are grateful to cardiothoracic surgeons of Moheb Hospital for their collaboration.

\section{Authors' Contributions}

Conception and design, collection of data, critical revision of the article, and administrative technical and scientific revision of the article: Mehrdad Mesbah kiaee. Conception and design, statistical analysis, and scientifically revision of the article: Saeid Safari. Conception and design, obtaining funding, and data interpretation: Gholamreza Movaseghi. Conception and design and statistical analysis: Mahmoudreza Mohaghegh Dolatabadi. Literature search, statistical analysis, and scientifically revision of the article: Masoud Ghorbanlo. Data collection, statistical analysis, writing the article, and critical revision of the article: Mohammad Mahdi Zamani.

\section{Funding/Support}

This study was funded by Research and Development Division of Moheb Hospital, Tehran, Iran.

\section{References}

1. Montes FR, Giraldo JC, Betancur LA, Rincon JD, Rincon IE, Vanegas MV, et al. Endotracheal intubation with a lightwand or a laryngoscope results in similar hemodynamic variations in patients with coronary artery disease. Can J Anaesth. 2003;50(8):824-8.

2. Puri GD, Marudhachalam KS, Chari P, Suri RK. The effect of magnesium sulphate on hemodynamics and its efficacy in attenuating the response to endotracheal intubation in patients with coronary artery disease. Anesth Analg. 1998;87(4):808-11.

3. Dube L, Granry JC. The therapeutic use of magnesium in anesthesiology, intensive care and emergency medicine: a review. Can J Anaesth. 2003;50(7):732-46.

4. Khalili G, Janghorbani M, Sajedi P, Ahmadi G. Effects of adjunct intrathecal magnesium sulfate to bupivacaine for spinal anesthesia: a randomized, double-blind trial in patients undergoing lower extremity surgery. J Anesth. 2011;25(6):892-7.

5. Mayer ML, Westbrook GL, Guthrie PB. Voltage-dependent block by $\mathrm{Mg} 2+$ of NMDA responses in spinal cord neurones. Nature. 1984;309(5965):261-3.

6. Magnesium sulphate as a novel treatment in resistant tic douloureux. In: Soleimanpour H, Ghafouri RR, Aghamohammadi D, Safari S, Marjani K, Soleimanpour M, et al., editors. Minerva anestesiologica; 2013 p. 1.

7. James MF, Beer RE, Esser JD. Intravenous magnesium sulfate inhibits catecholamine release associated with tracheal intubation. Anesth Analg. 1989;68(6):772-6.

8. Laurant P, Touyz RM, Schiffrin EL. Effect of magnesium on vascular tone and reactivity in pressurized mesenteric resistance arteries from spontaneously hypertensive rats. Can J Physiol Pharmacol.1997;75(4):293-300.

9. Jolliffe CT, Leece EA, Adams V, Marlin DJ. Effect of intravenous lidocaine on heart rate, systolic arterial blood pressure and cough responses to endotracheal intubation in propofol-anaesthetized dogs. Vet Anaesth Analg. 2007;34(5):322-30.

10. Mikawa K, Nishina K, Takao Y, Shiga M, Maekawa N, Obara H. Attenuation of cardiovascular responses to tracheal extubation: comparison of verapamil, lidocaine, and verapamil-lidocaine combination. Anesth Analg. 1997;85(5):1005-10.

11. Altura BT, Altura BM. Endothelium-dependent relaxation in coronary arteries requires magnesium ions. Br J Pharmacol. 1987;91(3):449-51

12. Sanders GM, Sim KM. Is it feasible to use magnesium sulphate as a hypotensive agent in oral and maxillofacial surgery? Ann Acad Med Singapore. 1998;27(6):780-5.

13. Wong $\mathrm{CH}$, Dey P, Yarmush J, Wu WH, Zbuzek VK. Nifedipineinduced analgesia after epidural injection in rats. Anesth Analg. 1994;79(2):303-6.

14. Do SH. Magnesium: a versatile drug for anesthesiologists. Korean JAnesthesiol. 2013;65(1):4-8.

15. Crozier TA, Radke J, Weyland A, Sydow M, Seyde W, Markakis E, et al. Haemodynamic and endocrine effects of deliberate hypotension with magnesium sulphate for cerebral-aneurysm surgery. EurJ Anaesthesiol.1991;8(2):115-21.

16. Elsharnouby NM, Elsharnouby MM. Magnesium sulphate as a technique of hypotensive anaesthesia. $\mathrm{Br} J$ Anaesth. 2006;96(6):727-31.

17. Nakaigawa Y, Akazawa S, Shimizu R, Ishii R, Ikeno S, Inoue S, et al. Effects of magnesium sulphate on the cardiovascular system, coronary circulation and myocardial metabolism in anaesthetized dogs. Br J Anaesth. 1997;79(3):363-8.

18. Moharari RS, Motalebi M, Najafi A, Zamani M, Imani F, Etezadi F, et al. Magnesium Can Decrease Postoperative Physiological Ileus and Postoperative Pain in Major non Laparoscopic Gastrointestinal Surgeries: A Randomized Controlled Trial. Anesth Pain Med. 2014; 4(1): e12750.

19. Shin YH, Choi SJ, Jeong HY, Kim MH. Evaluation of dose effects of magnesium sulfate on rocuronium injection pain and hemodynamic changes by laryngoscopy and endotracheal intubation. Korean J Anesthesiol. 2011;60(5):329-33.

20. Nastou H, Sarros G, Nastos A, Sarrou V, Anastassopoulou J. Prophylactic effects of intravenous magnesium on hypertensive emergencies after cataract surgery. A new contribution to the pharmacological use of magnesium in anaesthesiology. Magnes Res. 1995;8(3):271-6.

21. Delhumeau A, Granry J, Cottineau C, Bukowski J, Corbeau J, Moreau X. Comparative Vascular Effects of Magnesium Sulphate and Nicardipine during Cardiopulmonary Bypass. Ann Fr Anesth Re'anim. 15 edParis: RUE Javel,; 1995.

22. Ferasatkish R, Dabbagh A, Alavi M, Mollasadeghi G, Hydarpur E, Moghadam AA, et al. Effect of magnesium sulfate on extubation time and acute pain in coronary artery bypass surgery. Acta Anaesthesiol Scand. 2008;52(10):1348-52.

23. Yorukoglu D, Asik Y, Okten F. Rocuronium combined with i.v. lidocaine for rapid tracheal intubation. Acta Anaesthesiol Scand. 2003;47(5):583-7.

24. Pandey CK, Raza M, Ranjan R, Singhal V, Kumar M, Lakra A, et al. Intravenous lidocaine $0.5 \mathrm{mg} . \mathrm{kg}-1$ effectively suppresses fentanyl-induced cough. Can J Anaesth. 2005;52(2):172-5.

25. Hassani V, Movassaghi G, Goodarzi V, Safari S. Comparison of fentanyl and fentanyl plus lidocaine on attenuation of hemodynamic responses to tracheal intubation in controlled hypertensive patients undergoing general anesthesia. Anesth Pain Med. 2013;2(3):115-8.

26. Miller CD, Warren SJ. IV lignocaine fails to attenuate the cardiovascular response to laryngoscopy and tracheal intubation. $\mathrm{Br} J$ Anaesth.1990;65(2):216-9.

27. Singh H, Vichitvejpaisal P, Gaines GY, White PF. Comparative ef fects of lidocaine, esmolol, and nitroglycerin in modifying the hemodynamic response to laryngoscopy and intubation. J Clin Anesth. 1995;7(1):5-8.

28. Panda NB, Bharti N, Prasad S. Minimal effective dose of magnesium sulfate for attenuation of intubation response in hypertensive patients. J Clin Anesth. 2013;25(2):92-7.

29. Nooraei N, Dehkordi ME, Radpay B, Teimoorian H, Mohajeran SA. Effects of Intravenous Magnesium Sulfate and Lidocaine on Hemodynamic Variables Following Direct Laryngoscopy and Intubation in Elective Surgery Patients. Tanaffos. 2013;12(1). 